University of Wollongong

Research Online

Faculty of Engineering and Information

Faculty of Engineering and Information

Sciences - Papers: Part A

Sciences

$1-1-2014$

Collaborative learning through TaaS: a mobile system for courses over the cloud

Geng Sun

University of Wollongong, gs147@uowmail.edu.au

Jun Shen

University of Wollongong, jshen@uow.edu.au

Follow this and additional works at: https://ro.uow.edu.au/eispapers

Part of the Engineering Commons, and the Science and Technology Studies Commons

Research Online is the open access institutional repository for the University of Wollongong. For further information contact the UOW Library: research-pubs@uow.edu.au 


\title{
Collaborative learning through TaaS: a mobile system for courses over the cloud
}

\begin{abstract}
As mobile cloud-based learning gain wide acceptance, learners engaged in online courses have more opportunities to participate in virtual teams. In this paper we present a new system, Teamwork as a Service (TaaS), which is service-oriented and emphasizes on building a smart collaborative learning context in conjunction with cloudhosting learning management systems (LMSs). Each of the five services of TaaS aims to organize a certain type of refined learning activities, so as to join together to facilitate the whole collaborative learning process. The implementation details of TaaS are demonstrated, and some typical user interfaces (UIs) are illustrated about how TaaS works in real environments.
\end{abstract}

\section{Keywords}

courses, over, cloud, system, mobile, taas, learning, collaborative

Disciplines

Engineering | Science and Technology Studies

\section{Publication Details}

Sun, G. \& Shen, J. (2014). Collaborative learning through TaaS: a mobile system for courses over the cloud. 2014 IEEE 14th International Conference on Advanced Learning Technologies (ICALT 2014) (pp. 278-280). United States: IEEE. 


\title{
Collaborative Learning through TaaS: a Mobile System for Courses over the Cloud
}

\author{
Geng Sun, Jun Shen \\ Faculty of Engineering and Information Systems and Technology \\ University of Wollongong \\ Wollongong, Australia \\ Email: gs147@uowmail.edu.au,jshen@uow.edu.au
}

\begin{abstract}
As mobile cloud-based learning gain wide acceptance, learners engaged in online courses have more opportunities to participate in virtual teams. In this paper we present a new system, Teamwork as a Service (TaaS), which is service-oriented and emphasizes on building a smart collaborative learning context in conjunction with cloudhosting learning management systems (LMSs). Each of the five services of TaaS aims to organize a certain type of refined learning activities, so as to join together to facilitate the whole collaborative learning process. The implementation details of TaaS are demonstrated, and some typical user interfaces (UIs) are illustrated about how TaaS works in real environments.
\end{abstract}

Keywords-collaborative learning; web service; mobileaccessible system, implementation.

\section{INTRODUCTION}

Mobile cloud-based learning is thriving for its ease of use while the shortcomings of sole mobile devices [1]. To make the full use of mobile cloud-based learning, teachers involved in either on-campus or distance learning have shown great interests in the delivery of online courses, which could build virtual teams and adopt collaborative learning [2].

Teamwork in a mobile environment is significantly different from that in traditional learning. In this paper, we introduce an innovative approach to fill these gaps, seeking the optimal collaborative learning outcomes for learners. This approach is realized by the development of a serviceoriented mobile-accessible system, Teamwork as a Service (TaaS). Also, we implement TaaS over a cloud platform to make it come into real use in the online courses.

\section{MOTIVATION AND FRAMEWORK}

In our previous work [3], problems that may cause difficulties to learners who participate in collaborative learning in online courses are summarized. By the mean of service oriented architecture (SOA), and using Kolb's 'team learning experience' (KTLE) as the main concept [4], we orchestrate a teamwork-enhanced learning flow, which can be executed by our newly designed service-oriented and cloud-based system, TaaS, together with LMSs. TaaS consists of five web services, each of which takes one or more of the different modules in the KTLE to organize a certain type of activity in order to support learners to be more focused on teamwork, or to offer them computational choices to get into smart collaborative learning scenarios:
- For a mobile cloud-based course with team based assessment tasks, the Survey Service provides a platform for learners to know one another at the beginning of the collaborative learning, usually concurring with the release of the team assignment. Data about each learner's social features are collected, in terms of their learning styles and comprehensive teamwork skills set.

- $\quad$ The Jigsaw method introduced in [5] is commonly used for deepening learners' understanding of 'team purpose', the three stages of which can be imitated by the Jigsaw Service. It organizes a cloud Jigsaw Classroom containing different forms of meaningful personnel structures for learners to get into efficient discussion about their team assignments.

- The Bulletin Service allows learners to collaboratively define their 'team context' and 'team purpose', by writing down their thoughts about how to accomplish the team assignment in a task-subtask umbrella structure. It is also utilized to evaluate each pre-planned task's difficulty and learners' preference with regard to the task.

- We abolish the concentrated leadership and share leadership over the both sides of the system and learners. The duty to assign the suited 'team membership' for each capable team and allocate the clear-cut 'team role' for each team member is done by the Inference Service, which takes the responsibility to find out how to group learners into competitive teams. The reasoning process of team formation is supported by using a genetic algorithm (GA) method.

- Mutual supervision among learners is conducted by the Monitor Service. It works to regularize learners' behaviors during the 'team action' and 'team process'. In each team, each learner is assigned as the coordinator for another, a penalty mechanism, in addition, is embedded in this service.

\section{SYSTEM IMPLEMENTATION}

\section{A. System Implementation}

To execute a teamwork-enhanced learning flow for mobile cloud-based learning, we have composed TaaS with Moodle. Once an online course takes place, mobile learners 
get access to learning resources and perform their conventional learning activities through Moodle, whereas they utilize functions supported by TaaS to facilitate collaborative learning.

We have launched a Linux instance of the Amazon Elastic Cloud Computing (EC2) and have configured the server environment as Apache + PHP + Mysql, hosting system packages of TaaS and Moodle over it. TaaS can also work independently, so that it holds its own complete user registration and administration mechanisms. As shown in Fig. 1, we have integrated the five services introduced in Section II as a system and added a user-management module for controlling accounts.

The Usermanagement class holds the ability to run the necessary functions to control and administrate user accounts. By the SingleSignOn() method, the single-sign-on (SSO) technique is realized to enable users (teachers and learners) to $\log$ into TaaS if they already have valid Moodle accounts. We have created a new database of TaaS for storing teamwork-related data, such as learners' KLS capabilities, preferences, etc. The GetUserDataFromLms() method guarantees user data in both sides of LMS and TaaS are kept in synchronization all the time, to guarantee data consistency. Based on that, basic learning information are invoked from Moodle through its Web service APIs.

The SurveyService class gives teachers authorities to modify or reset the structure of surveys by themselves. Otherwise, results of five sets of questionnaires preset in this service are the default mechanism towards gathering the raw data about learners' characterizations [3]. We seek to use these questionnaires, one for each, to investigate five aspects of learners' features, namely four in Kolb's learning styles (KLS), i.e. diverging, accommodating, assimilating, and converging, and one in their comprehensive teamwork (CT) capabilities[6].

In the JigsawService class, web methods mainly function to organize the three-stage Jigsaw Classroom. Groups in each stage are formed with regard to a learner's best learning style in KLS [6].

The BulletinService class offers a wiki-like platform to establish a collaborative editing environment. It applies the CheckAuthority() to allow each published task to be modified by its publisher if authority matches, who is also required to mark each subtask's difficulty. Besides, nonpublisher learners can view and show their preference to each published task through SelectSubtaskPreference().

The InferenceService class is responsible for reasoning the suitable task allocation, by matching each learner to the most appropriate subtask and then grouping therefore competitive teams. It invokes raw data about learners and task/subtasks from the SurveyService class and BulletinService class, respectively. Then it conducts a computation using GA. The details of GA can be referred to another of our work [7]. GA can be triggered by teachers in one of the two ways oriented to two different collaborative scenarios: 'Keeping the balance between each team' and
'Letting the learners show their capabilities in the best possible way' [3] [7].

In the MonitorService class, the SetCoordinator() method runs initially, then each learner is in position. A learner is linked with his/her coordinator by a file transmission channel.

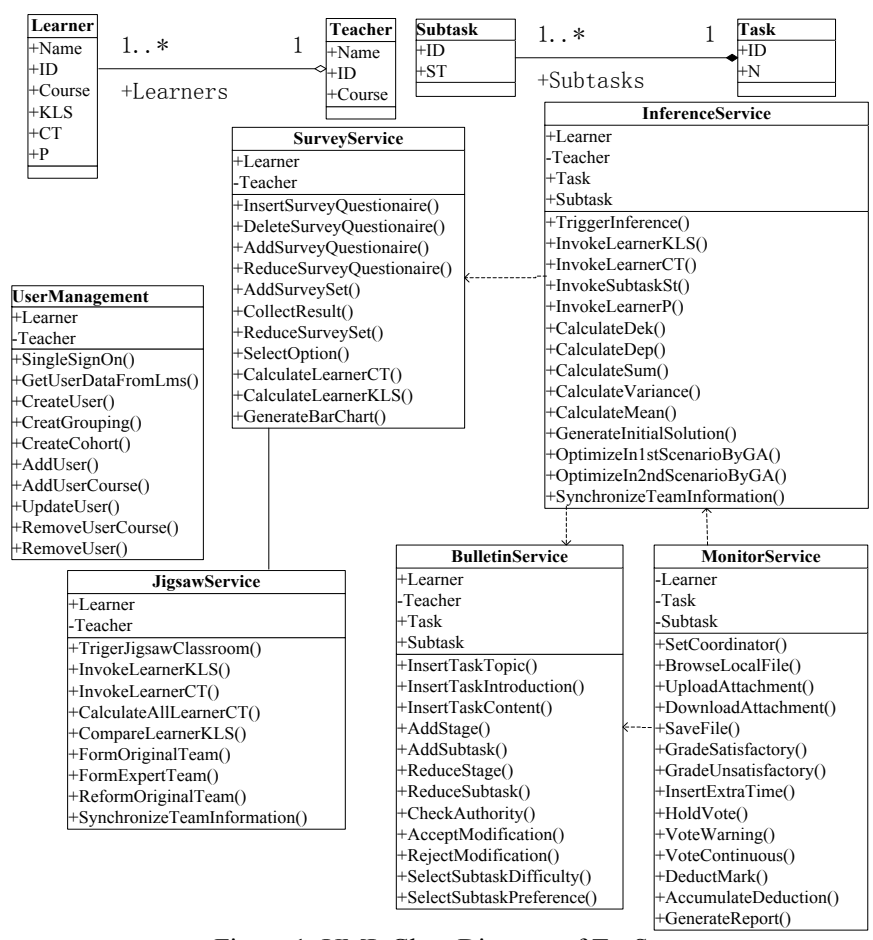

Figure 1. UML Class Diagram of TaaS

\section{B. User Interface}

The UI of teachers' main page of TaaS is shown in Fig. 2. Teachers can click buttons to launch several events, such as starting each stage of the Jigsaw Classroom. They also have authorities to change the structure of surveys, and preset the penalty mechanisms embedded in the Monitor Service (set the maximum times allowed for a learner to get 'unsatisfactory' grade, the deduction weight for each 'unsatisfactory' and 'warning' or percentage of final mark given by teacher if applicable).

Teachers can organize the Jigsaw Classroom at any stage by choosing an option in the dropdown list. The three stages can be timed and set in automatic operation for which a button is placed. They can activate grouping as well by triggering the Inference Service in either way, for which a dropdown list has also been provided. They can also check the team formation by clicking the corresponding button.

The UI of learners' main page is shown as Fig. 3. A learner's capabilities in five aspects are summarized in a bar chart, and can be checked by their teammates. S/he can click buttons to participate in learning activities by entering new pages. For example, the "Participate in survey" button is designed for showing a learner the interface of answering the five sets of questionnaires pre-installed in the Survey Service. His/her team information and task information are shown at the bottom of the main page. 


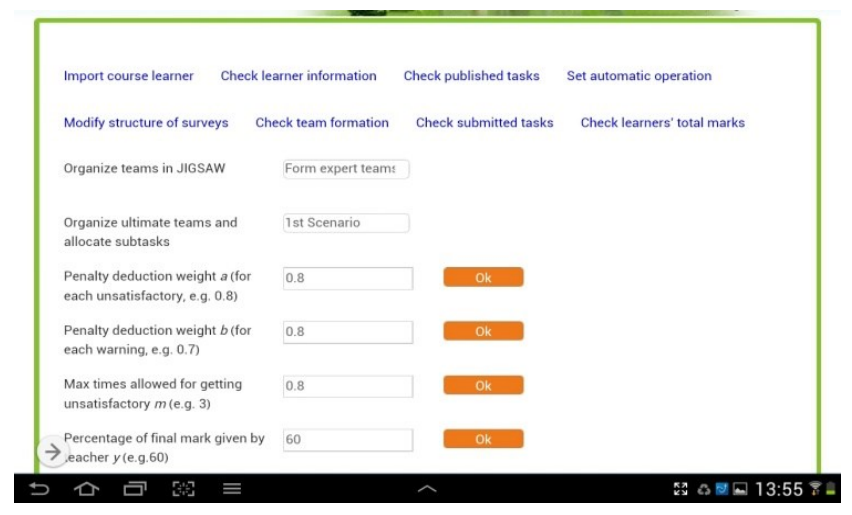

Figure. 2 Main Page of the Teacher User

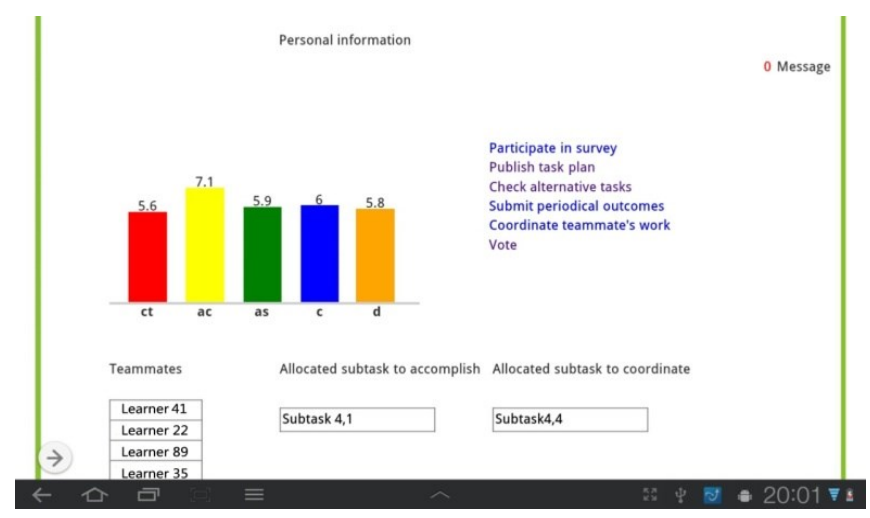

Figure 3. Main Page of the Learner User

A learner can click "Publish task plan" or "Check alternative tasks" buttons to use the Bulletin Service, entering new pages to fill the related contents in the provided textboxes. While they are planning schedules, the structure of tasks is scalable, by adding or reducing subtasks and the stages of subtasks. Based on a learner's authority for a task, s/he can also set its subtasks' difficulties in KLS when publishing and his/her preferences when checking.

As a performer of a subtask, a learner can click the "Submit periodical outcomes" button to obtain an upload link for submitting periodical outcomes, which are transferred directly to his/her coordinator. On the other hand, as a coordinator of one of his/her teammates, a learner can click the "Coordinate teammate's work" button to deal with this job. In a subsequent page, s/he can download the work submitted from the subtasks' performer who $s /$ he is in charge of, and grade it accordingly. The 'satisfactory' and 'unsatisfactory' buttons correspond to web methods in the MonitorService class (see Fig. 1).

If an 'unsatisfactory' grade is given, the coordinator is required to decide how much 'extra time' should be given for work revision. A new message is sent to the performer when the 'extra time' ends, at which time the revised work must be resubmitted. Then the coordinator judges it again. Furthermore, if a team member' in-progress work receives 'unsatisfactory' for more than the allowed maximum times preset by the teacher, a vote is held inside a team. In the same way, the other team members can click the "Vote" button on their own main pages to express their decision, by choosing "warning" or "continue" in the subsequent pages to call relevant web methods in the MonitorService class (see Fig. 1).The example UI for processing mutual supervision is shown as in Fig. 4. The status of the message box changes when any new announcement arrives or any new activity occurs, which commonly notify what should be done in the next steps of team learning activity.

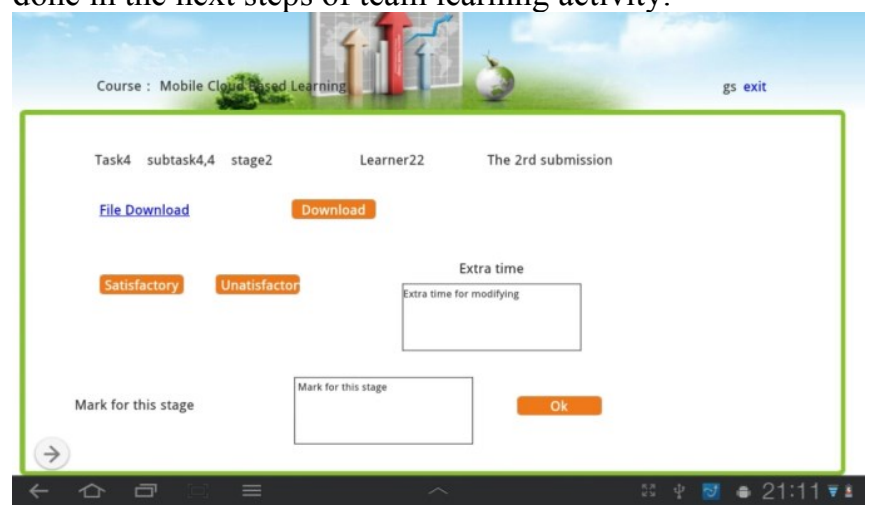

Figure 4. Example UI of Processing Mutual Supervision

\section{CONCLUSION}

In this paper, we introduced the implementation of a service-oriented system, TaaS. Using these web services through mobile devices, learners are able to deepen their understanding of team learning purpose, practice their planning capabilities, participate in competitive teams with suitable task allocation and supervise other team members to avoid delays and guarantee efficiency. Details about implementation of TaaS are given to show how this system works at the backend, and we demonstrate some typical UIs.

\section{REFERENCES}

[1] N. M. Rao, "Cloud Computing Through Mobile-Learning", International Journal of Advanced Computer Science and Applications, vol. 1, no. 6, pp. 42-47, 2010

[2] J. Liao and M. Wang, "A Collaborative Learning System Based on Cloud and E-Commerce", $8^{\text {th }}$ IEEE International Conference on eBusiness Engineering (ICEBE), pp.16-23, Beijing, China, Octorber, 2011.

[3] G. Sun and J. Shen, "Teamwork as a Service: a Cloud-based System for Enhancing Teamwork Performance in Mobile Learning", The $13^{\text {th }}$ IEEE International Conference on Advanced Learning Technologies (ICALT), pp. 376-378, Beijing, China, July 2013.

[4] A. B. Kayes, D. C. Kayes and D. A. Kolb, 'Developing teams using the Kolb team learning experience'. Simulation \& Gaming, vol. 36, pp. 355-363, 2005.

[5] E. Aronson, N. Blaney, C. Steophan, J. Sikes and M. Snapp, The Jigsaw Classroom, Beverly Hills, CA, USA, 1978.

[6] A. Y. Kolb and A. D. Kolb, 'Learning styles and learning spaces: Enhancing experiential learning in higher education'. Academy of Management Learning and Education. vol.4, no.2, pp. 193-212, 2005.

[7] G. Sun and J. Shen, "Facilitating Collaborative Learning in TaaS: a Mobile Cloud System for Enhancing Teamwork Performance", The IEEE International Conference on Systems, Man and Cybernetics (SMC), pp. 681-686, Manchester, UK, October 2013. 